\title{
Shape and stability of a viscous thread
}

\author{
Bohr, Tomas; Senchenko, Sergey
}

Published in:

Physical Review E

Link to article, DOI:

10.1103/PhysRevE.71.056301

Publication date:

2005

Document Version

Publisher's PDF, also known as Version of record

Link back to DTU Orbit

Citation (APA):

Bohr, T., \& Senchenko, S. (2005). Shape and stability of a viscous thread. Physical Review E, 71(5), 056301. https://doi.org/10.1103/PhysRevE.71.056301

\section{General rights}

Copyright and moral rights for the publications made accessible in the public portal are retained by the authors and/or other copyright owners and it is a condition of accessing publications that users recognise and abide by the legal requirements associated with these rights.

- Users may download and print one copy of any publication from the public portal for the purpose of private study or research.

- You may not further distribute the material or use it for any profit-making activity or commercial gain

- You may freely distribute the URL identifying the publication in the public portal

If you believe that this document breaches copyright please contact us providing details, and we will remove access to the work immediately and investigate your claim 


\title{
Shape and stability of a viscous thread
}

\author{
Sergey Senchenko ${ }^{1,2, *}$ and Tomas Bohr ${ }^{1}$ \\ ${ }^{1}$ Physics Department, Danish Technical University, DK-2800 Lyngby, Denmark \\ ${ }^{2}$ Optics and Fluid Dynamics Department, Ris $\phi$ National Laboratory, DK-4000 Roskilde, Denmark
}

(Received 1 March 2004; revised manuscript received 18 November 2004; published 3 May 2005)

\begin{abstract}
When a viscous fluid, like oil or syrup, streams from a small orifice and falls freely under gravity, it forms a long slender thread, which can be maintained in a stable, stationary state with lengths up to several meters. We discuss the shape of such liquid threads and their surprising stability. The stationary shapes are discussed within the long-wavelength approximation and compared to experiments. It turns out that the strong advection of the falling fluid can almost outrun the Rayleigh-Plateau instability. The asymptotic shape and stability are independent of viscosity and small perturbations grow with time as $\exp \left(\mathrm{C} t^{1 / 4}\right)$, where the constant is independent of viscosity. The corresponding spatial growth has the form $\exp \left[(z / L)^{1 / 8}\right]$, where $z$ is the down stream distance and $L \sim Q^{2} \sigma^{-2} g$ and where $\sigma$ is the surface tension divided by density, $g$ is the gravity, and $Q$ is the flux. We also show that a slow spatial increase of the gravitational field can make the thread stable.
\end{abstract}

DOI: 10.1103/PhysRevE.71.056301

PACS number(s): 47.20.Gv, 47.20.Dr, 47.54.+r

\section{INTRODUCTION}

When honey or syrup is poured from an outlet, one easily generates very long threads of flowing fluid of surprising beauty and stability. A uniform column of fluid is unstable due to surface tension effects- the famous Rayleigh-Plateau instability [1]. Viscosity diminishes the strength, but does not remove the instability, and thus the observation of stable falling viscous threads of, say, $2 \mathrm{~m}$ is surprising-in our local stairwell, we have seen threads of syrup up to around $10 \mathrm{~m}$. In the present paper we shall discuss the shape and stability of such falling viscous jets or threads. We should note from the outset that we are confining our attention to Newtonian fluids (e.g., syrup or silicone oil).

Our starting point in Sec. II is the long-wave approximation (see, e.g., [2]), which only takes into account the leading-order dependence of the velocity field on the radial variable. In Sec. III we study the stationary solutions and, in particular, their asymptotic forms. The final asymptotics (for large downstream distance $z$ ) is always governed solely by gravity, as in a free fall. We perform a simple experiment with heavy silicone oil and find that the observed stationary shape of a thread is in a good agreement with the theoretical prediction (Sec. III). Then we proceed with a linear stability analysis (Sec. IV). After a recapitulation of the classical Rayleigh-Plateau instability in the long-wavelength limit in the absence of gravity, we study the full linear stability problem of a falling thread using a Lagrangian description and solve it asymptotically.

\section{DERIVATION OF THE MODEL}

To describe the dynamics of a fluid column falling under gravity, we assume that the flow is axisymmetric and that the velocity has only a radial and a vertical component. In the long-wave approximation $[3,4]$ the velocity is expanded in a

\footnotetext{
*Electronic address: senchen@fysik.dtu.dk
}

power series in the radial variable $r$ and one finds (expressing mass conservation and the $z$ component of the NavierStokes equation, respectively)

$$
\begin{gathered}
\left(h^{2}\right)_{t}+\left(w h^{2}\right)_{z}=0, \\
w_{t}+w w_{z}=-\frac{\gamma}{\rho}(\kappa)_{z}+g+3 \nu \frac{\left(w_{z} h^{2}\right)_{z}}{h^{2}}
\end{gathered}
$$

[given as Eqs. (17) and (18) in [4]], where $w(z, t)$ is the leading-order term in the expansion of the vertical velocity field in $r, h(z, t)$ is the radius of the jet, $\gamma$ is the surface tension coefficient, $\nu$ is the kinematic viscosity, and $g$ is the gravitational acceleration. The curvature term in Eq. (2) is

$$
\kappa=\left(\sqrt{1+h_{z}^{2}}\right)^{-1}\left(\frac{1}{h}-\frac{h_{z z}}{1+h_{z}^{2}}\right),
$$

but since we are interested in the asymptotic properties of thin threads, we neglect the curvature in the $(r, z)$ plane compared to the one around the axis of the thread and assume that $h_{z} \ll 1$. Thus we shall, throughout the paper, use the slender approximation

$$
\kappa \approx \frac{1}{h} .
$$

We now introduce dimensionless variables through

$$
z \rightarrow \alpha z, \quad t \rightarrow \beta t \quad h \rightarrow \alpha h, \quad w \rightarrow \frac{\alpha}{\beta} v,
$$

where $\alpha$ and $\beta$ are dimensional coefficients. Thus Eq. (2) acquires the following form (with $\sigma=\gamma / \rho$ ):

$$
v_{t}+v v_{z}=\frac{\beta^{2}}{\alpha} g-\frac{\beta^{2}}{\alpha^{3}} \sigma\left(\frac{1}{h}\right)_{z}+3 \nu \frac{\beta}{\alpha^{2}} \frac{\left(v_{z} h^{2}\right)_{z}}{h^{2}},
$$

whereas Eq. (1) preserves its form since it is homogeneous in space and time variables.

We choose $\alpha$ and $\beta$ such that the two first coefficients on the right-hand side (RHS) of Eq. (6) are equal to unity: i.e., 


$$
\alpha=\sigma^{1 / 2} g^{-1 / 2}, \quad \beta=\sigma^{1 / 4} g^{-3 / 4} .
$$

This allows us to consider both viscid and inviscid cases by means of the last coefficient, the dimensionless viscosity $\Gamma$ $=3 \nu \beta / \alpha^{2}=3 \nu \sigma^{-3 / 4} g^{1 / 4}$. [The Morton number often used in the bubble literature is $(\Gamma / 3)^{4}$ and the Kapitza number used for falling films is $\mathrm{Ka}=(\Gamma / 3)^{-4 / 3}$.] Note that this choice of rescaling means that lengths are measured in units of the capillary length $l_{c}=\sigma^{1 / 2} g^{-1 / 2}=\alpha$. Thus the nondimensionalized model has the following form:

$$
\begin{gathered}
\left(h^{2}\right)_{t}+\left(v h^{2}\right)_{z}=0, \\
v_{t}+v v_{z}=-\left(\frac{1}{h}\right)_{z}+1+\Gamma \frac{\left(v_{z} h^{2}\right)_{z}}{h^{2}} .
\end{gathered}
$$

Note that it is possible to eliminate the parameter $\Gamma$ from Eqs. (8) and (9) by the following scaling:

$$
z \rightarrow \Gamma^{2 / 3} z, \quad t \rightarrow \Gamma^{1 / 3} t, \quad h \rightarrow \Gamma^{-2 / 3} h, \quad v \rightarrow \Gamma^{1 / 3} v
$$

Although the scaling (10) allows us to make the system parameter free, we shall, in the following, retain $\Gamma$ and stick to the scaling (5)-(7), since we want to be able to trace independently the effects produced by physical constants (like the scaled viscosity $\Gamma$ ) and experimentally controlled parameters (like flux $q$ ). Second, the scaling (10) becomes ill defined in the limit $\Gamma \rightarrow 0$, which is the case that we shall deal with in Sec. IV.

\section{STATIONARY SOLUTIONS}

The shape of a stationary thread has been studied by several authors (see [5-10]), but since the results are somewhat scattered and incomplete, we have found it important to describe the stationary states in some detail. For stationary solutions the nondimensional flux $q=h^{2} v$ is constant and we end up with the following equation for the velocity field only:

$$
v v_{z}=1-\frac{v_{z}}{2 \sqrt{q v}}+\Gamma v_{z z}-\Gamma \frac{v_{z}^{2}}{v} .
$$

The flux $q$ is by the scaling (7) related to the physical flux $Q$ as

$$
q=\alpha^{-3} \beta Q / \pi=\sigma^{-5 / 4} g^{3 / 4} Q / \pi .
$$

When Eq. (11) is solved forward in $z$-i.e., as an "initialvalue problem" - the typical solutions will diverge for large $z$. This can be circumvented by integrating backwards noting that the fixed point $\left(v, v_{z}\right)=(0,0)$ has a well-defined unstable manifold (separating solutions that diverge to plus or minus infinity), which upon backward integration becomes a stable manifold. In Fig. 1 we show typical phase-space trajectories found by solving Eq. (11) numerically by means of a fourthorder Runge-Kutta method, starting from "initial conditions" $\left(v_{0}, v_{z 0}\right)$ at large $z$ and integrating backwards. It is seen that the dependence on the particular choice of downflow conditions is very weak since all phase trajectories quickly con-

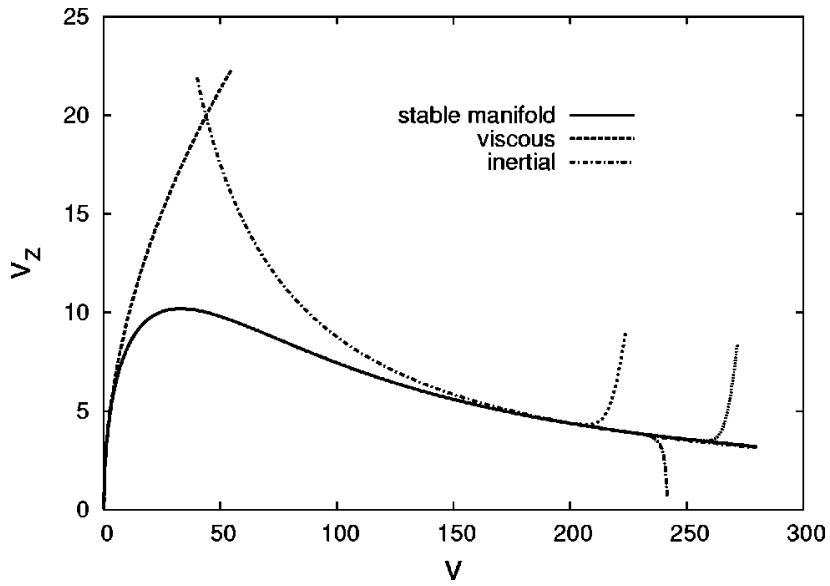

FIG. 1. The phase plane for Eq. (11) with $\Gamma=40$ and $q=1$. It is seen that, upon backward integration, trajectories quickly converge to a well-defined "unstable manifold" (solid line) for the fixed point $\left(v, v_{z}\right)=(0,0)$. The asymptotic solution for large $z, v \sim \sqrt{z}$, is shown as the dot-dashed line and is governed by inertia and gravity. The asymptotic solution for small $z, v \sim z^{2}$, is shown as the dashed line and is obtained by neglecting inertia.

verge to the well-defined stable manifold. Thus, even for a thread of moderate length the shape is uniquely determined irrespective of the precise downstream conditions, just as we would expect.

The asymptotic behavior of the solution as $z \rightarrow \infty$ is easily seen to be controlled by only the two first terms in Eq. (11): i.e.,

$$
v v_{z}=1
$$

giving

$$
\begin{gathered}
v=\sqrt{2 z}, \\
h=\sqrt{\frac{q}{v}}=q^{1 / 2}(2 z)^{-1 / 4} .
\end{gathered}
$$

This asymptotic solution is shown by the dot-dashed curve in Fig. 1 (marked "inertial").

The behavior of the unstable manifold near the fixed point $\left(v, v_{z}\right)=(0,0)$ can be found by expanding in $z$. Clearly we must take $v=C z^{2}+O\left(z^{3}\right)$ for the RHS of Eq. (11) to remain finite as $z \rightarrow 0$. Inserting this expression into Eq. (11), we see that the inertial term $v v_{z}$ can be neglected, since it contributes only as $z^{3}$, whereas all other terms contribute with $z^{0}$ terms, and we find

$$
1-\sqrt{C / q}-2 \Gamma C=0
$$

with the (positive) solution

$$
C=\frac{1+4 \Gamma q-\sqrt{1+8 \Gamma q}}{8 \Gamma^{2} q} .
$$

With this choice of $C$ the solution

$$
v=C z^{2},
$$




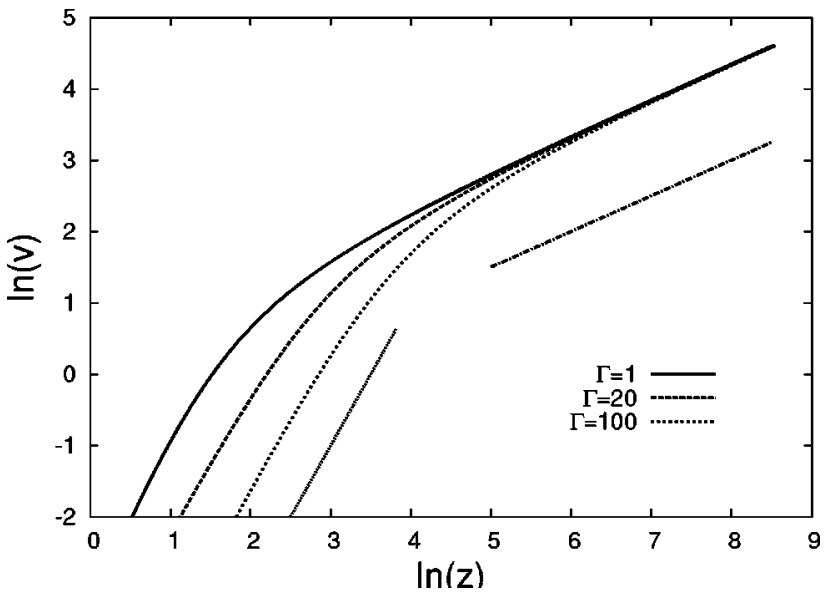

FIG. 2. Plot of the numerical solution of Eq. (11) for different values of $\Gamma$ and $q=1$. The viscous (18) and inertial (14) asymptotic are shown, respectively, by the straight lines.

$$
h=\sqrt{\frac{q}{C}} z^{-1}
$$

is in fact an exact solution to Eq. (11), when the inertial term $v v_{z}$ is neglected. This $v(z)$ is shown by the dotted curve in Fig. 1 (marked "viscous").

If in Eq. (16) we neglect the surface tension effects-i.e., consider the $\Gamma q \gg 1$ for the coefficient $C$-we get

$$
C \approx \frac{1}{2 \Gamma} .
$$

The crossover between the viscous and inertial solutions is roughly given by the value $z^{*}$ where they become equal: i.e.,

$$
z^{*} \approx\left(\frac{\sqrt{2}}{C}\right)^{2 / 3} \sim \Gamma^{2 / 3}
$$

for $\Gamma q \gg 1$. In Fig. 2 we plot $v(z)$ for various values of $\Gamma$.

\section{Experimental observation of a stationary state}

We have performed experiments to investigate the form of falling threads. We shall describe them briefly here to show that one finds good agreement with the results of the previous section. More details on the setup and data analysis can be found in [11]. The experimental setup consists of a cylindrical tank filled with an experimental fluid. The outlet at the bottom of the tank is controlled by a removable plug. When the liquid exits from the upper tank, the stream is collected in the lower tank. To avoid disturbances from external air flows we shelter the installation with the metal sheets. In our experiments we used heavy silicone oil with $\mu=60 \mathrm{Pas}, \rho$ $=1 \mathrm{~g} \mathrm{~cm}^{-3}$, and $\sigma=20 \mathrm{~cm}^{3} \mathrm{~s}^{-2}$, which corresponds to $\Gamma$ $\approx 1000$.

The flow was filmed with a video camera and the thread profile was found from a sequence of separate runs, each recording a small segment with a length of around $4 \mathrm{~mm}$, with a distance of $10 \mathrm{~cm}$, made under identical conditions.

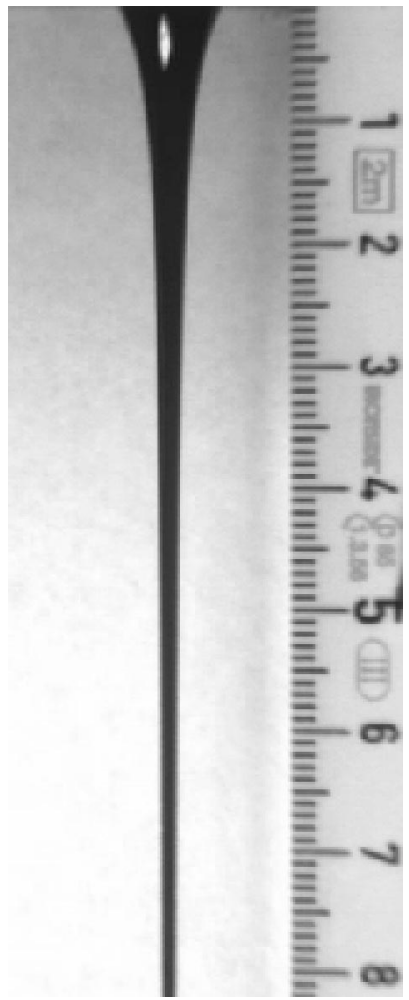

FIG. 3. General view of a viscous thread, $8 \mathrm{~cm}$ down from the outlet.

Figure 3 shows the upper $8 \mathrm{~cm}$ of the thread and Fig. 4 shows one of the 3-mm segments - appearing cylindrical on this short scale.

Experimental results for two different fluxes are given in Figs. 5 and 6 where we also compare with numerical solu-

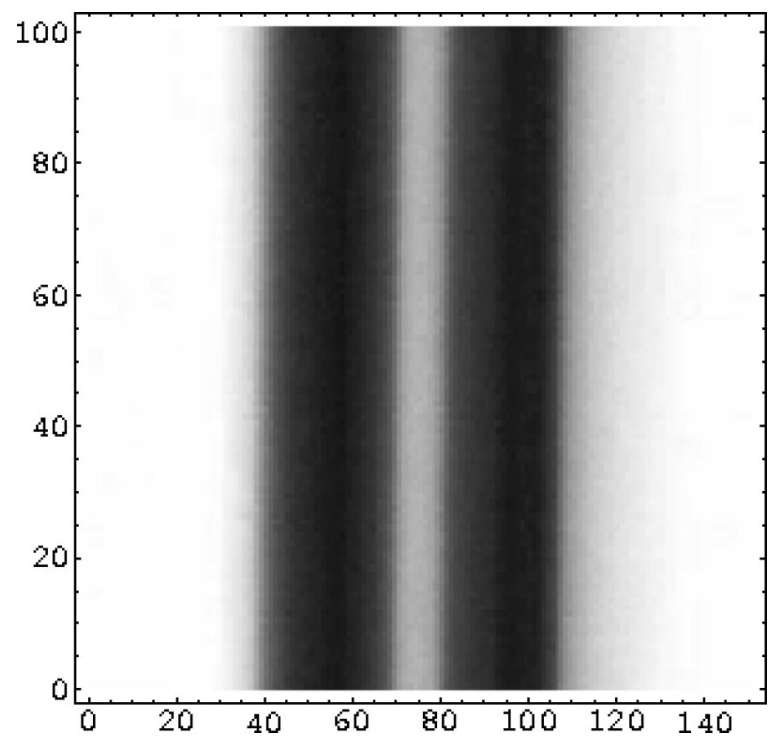

FIG. 4. Typical segment of a thread, photographed by chargecoupled-device (CCD) camera. The thread is seen as the dark black area. The vertical bands of different shadings are caused by the illumination. The frame shown is $4 \mathrm{~mm}$ high and $5 \mathrm{~mm}$ wide. The edge of the thread is estimated as the contour level with $95 \%$ of the brightness of the background. 


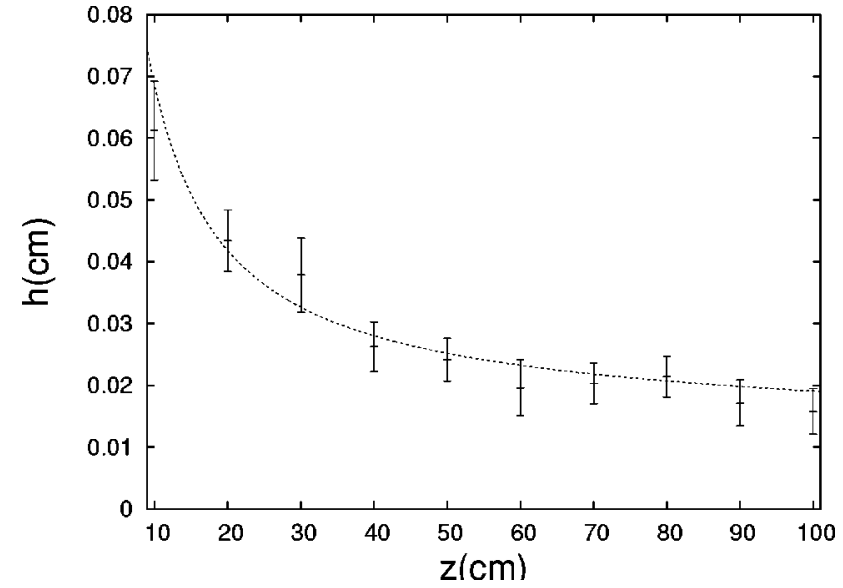

FIG. 5. Experimental data points (with error bars) vs numerical solution for a stationary thread, $Q=0.46 \mathrm{~cm}^{3} / \mathrm{s}, q=0.65$.

tions. The numerical solutions for the stationary profile were chosen such that they match the data at the outlet [i.e., $h(z$ $=0)=0.46 \mathrm{~cm}]$ which is outside of the figure. This might seem somewhat unphysical, since the long-wavelength approximation does not apply at the outlet. Alternatively one can, as seen from the figures, choose to fit at $z=10 \mathrm{~cm}$ (leftmost point in the figures) with similar results. The crossover length $z^{*}$ is comparable with the length of the thread, so we only see the beginning of the asymptotic regime (14). With our setup the thread remains perfectly continuous without any drop formation under constant flux conditions, even down to the lowest controllable fluxes, where the thread is as thin as a spider's web. The same is true when we increase the thread length to $3 \mathrm{~m}$.

\section{STABILITY PROPERTIES}

\section{A. Stability of a fluid cylinder in the long-wave length approximation}

To set the stage for the stability analysis, we first reproduce the classical Rayleigh-Plateau instability in the longwave length approximation $[1,2]$. The starting point is the (dimensional) set of equations (1) and (2). In the absence of gravity $(g=0)$ the stationary state is a cylinder moving with constant velocity. We thus assume

$$
\begin{aligned}
& v=v_{0}+\widetilde{v}(z, t), \\
& h=h_{0}+\widetilde{h}(z, t),
\end{aligned}
$$

and obtain the linearized system

$$
\begin{gathered}
\widetilde{v}_{t}+v_{0} \widetilde{v}_{z}=\sigma \frac{\widetilde{h}_{z}}{h_{0}^{2}}+\nu \widetilde{v}_{z z}, \\
\widetilde{h}_{t}+v_{0} \widetilde{h}_{z}=-\frac{1}{2} h_{0} \widetilde{v}_{z} .
\end{gathered}
$$

It is convenient to go to the comoving frame

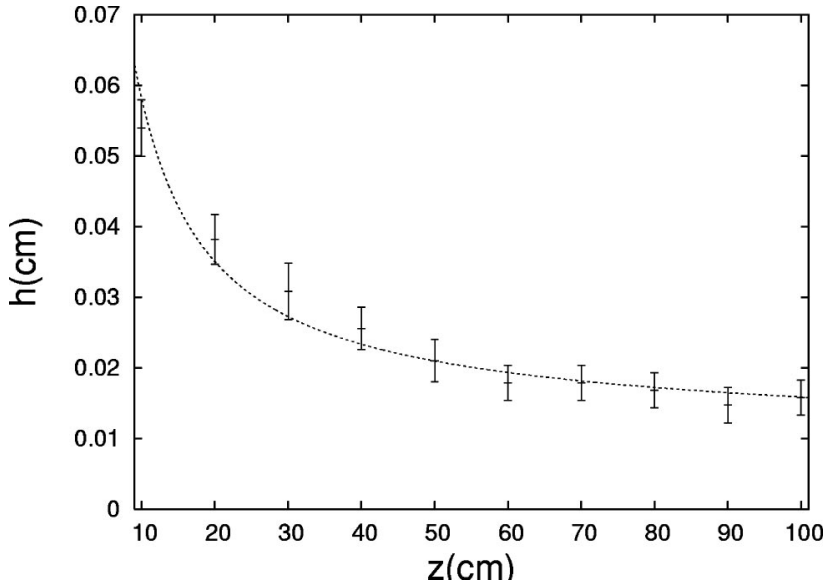

FIG. 6. Experimental data points (with error bars) vs numerical solution for a stationary thread, $Q=0.32 \mathrm{~cm}^{3} / \mathrm{s}, q=0.45$.

$$
y=z-v_{0} t, \quad \tau=t,
$$

where

$$
\begin{gathered}
\widetilde{v}_{\tau}=\sigma \frac{\tilde{h}_{y}}{h_{0}^{2}}+\nu \widetilde{v}_{y y}, \\
\tilde{h}_{\tau}=-\frac{1}{2} h_{0} \widetilde{v}_{y} .
\end{gathered}
$$

Transforming as usual to Fourier modes as

$$
(\tilde{v}, \tilde{h})=\left(C_{1}, C_{2}\right) \exp (i k y+s \tau)
$$

leads to the dispersion relation

$$
s_{ \pm}=\frac{1}{2}\left[-\nu k^{2} \pm \sqrt{\frac{2 \sigma k^{2}}{h_{0}}+\nu^{2} k^{4}}\right],
$$

which, within the long-wave length region $k h_{0} \ll 1$ coincides with the well-known results for the classical RayleighPlateau instability [1].

In the long-wave length limit $k^{2} \ll \sigma /\left(\nu^{2} h_{0}\right)$ the dynamics is inviscid and given by (for the unstable mode with positive s)

$$
s=k \sqrt{\frac{\sigma}{2 h_{0}}} .
$$

\section{B. Stability of the thread solution}

We are now ready to study the stability of the spatially varying thread solution-i.e., the stability of the stationary states of (8) and (9):

$$
\begin{gathered}
h_{t}^{2}+\left(v h^{2}\right)_{z}=0 \\
v_{t}+v v_{z}=-\left(\frac{1}{h}\right)_{z}+1+\Gamma \frac{\left(v_{z} h^{2}\right)_{z}}{h^{2}} .
\end{gathered}
$$

We linearize around the stationary solution $\left(v_{0}(z), h_{0}(z)\right)$, given in Eqs. (14) and (15) as 


$$
\begin{aligned}
& v=v_{0}(1+a), \\
& h=h_{0}(1+b),
\end{aligned}
$$

to obtain the linear system

$$
\begin{gathered}
a_{t}+a_{z}\left(v_{0}-\frac{3 \Gamma}{2 v_{0}} v_{0 z}\right)+a v_{0}^{-1} v_{0 z}\left(2 v_{0}-\Gamma v_{0 z}^{-1} v_{0 z z}+\frac{\Gamma}{2 v_{0}} v_{0 z}\right) \\
-\Gamma a_{z z}-b_{z}\left(q^{-1 / 2} v_{0}^{-1 / 2}+\Gamma v_{0}^{-1} v_{0 z}\right)-\frac{1}{2} b q^{-1 / 2} v_{0}^{-3 / 2} v_{0 z}=0 \\
b_{t}+v_{0} b_{z}+\frac{v_{0}}{2} a_{z}=0
\end{gathered}
$$

To get rid of the advection term, we introduce the stretched spatial variable $y$ as

$$
y=\int \frac{d z}{v_{0}(z)},
$$

so that $v_{0}(z) \partial_{z}=\partial_{y}$. We also define the function $W(y)$ as

$$
W(y)=v_{0}(z(y)),
$$

and these definitions transform Eqs. (32) and (33) into

$$
\begin{aligned}
a_{t}+a_{y}+2 W^{-1} W_{y} a= & q^{-1 / 2} W^{-3 / 2} b_{y}+\frac{q^{-1 / 2}}{2} W^{-5 / 2} W_{y} b \\
& +\Gamma W^{-2}\left[a_{y y}+2 W^{-1} W_{y} b_{y}\right. \\
& \left.+\left(W_{y y}-2 W^{-2} W_{y}^{2}\right) a\right], \\
b_{t} & +b_{y}+\frac{a_{y}}{2}=0 .
\end{aligned}
$$

For the inertial stationary solution $v_{0}(z)=\sqrt{2 z}$ we have explicitly

$$
z=\frac{y^{2}}{2}, \quad W(y)=y,
$$

and we finally transform Eqs. (36) and (37) into the comoving frame of reference,

$$
y=x+t, \quad t=t,
$$

to obtain

$$
\begin{gathered}
b_{t}+\frac{a_{x}}{2}=0 \\
a_{t}+2 a(x+t)^{-1}=q^{-1 / 2}(x+t)^{-3 / 2} b_{x}+\frac{1}{2} q^{-1 / 2}(x+t)^{-5 / 2} b \\
+\Gamma(x+t)^{-2}\left[a_{x x}+2(x+t)^{-1} b_{x}\right. \\
\left.-2(x+t)^{-2} a\right] .
\end{gathered}
$$

We now Fourier transfom in $x$, assuming that the asymptotic behavior will not be influenced by the slow algebraic variation with $x$ in the denominators as long as $t \gg x$, an assump- tion which will be verified in the Appendix. Thus we find, in terms of the Fourier transforms $\tilde{a}(k, t)$ and $\tilde{b}(k, t)$,

$$
\tilde{a}=\frac{2 i}{k} \tilde{b}_{t},
$$

$$
\begin{aligned}
\tilde{a}_{t} \approx & -2 \tilde{a} t^{-1}+i k q^{-1 / 2} t^{-3 / 2} \tilde{b}+\frac{1}{2} q^{-1 / 2} t^{-5 / 2} \tilde{b} \\
& +\Gamma t^{-2}\left(-k^{2} \tilde{a}+2 i t^{-1} k \tilde{b}-2 t^{-2} \tilde{a}\right) .
\end{aligned}
$$

As one can see immediately from the structure of Eq. (43), all viscous terms are subdominant with respect to surface tension terms in the limit $t \mapsto \infty$. In this limit we obtain the equations

$$
\begin{gathered}
\tilde{a}=\frac{2 i}{k} \tilde{b}_{t}, \\
\tilde{a}_{t}=-2 \widetilde{a} t^{-1}+i k q^{-1 / 2} t^{-3 / 2} \tilde{b} .
\end{gathered}
$$

Making finally the substitution

$$
\tilde{b}=t^{-1} B,
$$

we find

$$
B_{t t}=\frac{k^{2} q^{-1 / 2}}{2} t^{-3 / 2} B .
$$

The WKB ansatz $[12,13]$

$$
B(k, t)=B_{0} \exp \left(\int^{t} \Phi\left(k, t^{\prime}\right) d t^{\prime}\right)
$$

gives

$$
B_{+}(t)=B_{0} \exp \left(2 \sqrt{2} k q^{-1 / 4} t^{1 / 4}\right)
$$

The WKB approximate (49) is asymptotically valid for $t \mapsto \infty$ since $\Phi(k, t) \propto t^{-3 / 2}$, which decays sufficiently rapidly (see [12]).

If we assume that the amplitude $B_{0}(k)$ is localized around a particular wave vector $k_{0}$, i.e., as a Gaussian

$$
B_{0}(k)=\exp \left[-c\left(k-k_{0}\right)^{2}\right]
$$

we find that the maximum of the envelope, in the comoving frame, grows as

$$
B(x=0) \sim \exp \left(2 q^{-1 / 2} t^{1 / 2} / c\right)
$$

for sufficiently large $t$ or, equivalently,

$$
B\left(z=t^{2} / 2\right) \sim \exp \left(2^{5 / 4} q^{-1 / 2} z^{1 / 4} / c\right)
$$

for the perturbation advected along the thread. Thus the typical instability time is $t_{c} \sim q$ and from Eqs. (12) and (7) we can estimate the typical (dimensional) instability length-in time and in space, respectively_assuming that $c \sim O(1)$ :

$$
\begin{gathered}
T_{c} \sim Q \sigma^{-1}, \\
Z_{c} \sim g t_{c}^{2} \sim Q^{2} g \sigma^{-2} .
\end{gathered}
$$

One can understand the main result of this section from 
simple physical considerations: namely, using a stretching argument $[13,14]$. A slice of fluid of length $l(t)$ is advected by the flow and stretched: $d l / d t=l \partial v / \partial z$. For the inertiadominated solution $v(z)=\sqrt{2 z}$ we have $d l / d t=l / \sqrt{2 z}$. From the above equation and the free-fall dynamics of the fluid element (i.e., $z=t^{2} / 2$ ) it immediately follows that $d l / d t=l / t$ and $l(t)=l_{0} t / t_{0}$. Hence, the fluid element stretches like $t$, and the same is true for the wavelength of the initial perturbation: $\lambda(t)=\lambda_{0} t / t_{0}$. Consequently, the wave vector stretches as $k(t)=k_{0} t_{0} / t$. From Eq. (15) we have $h(t) \propto q^{1 / 2} t^{-1 / 2}$ and thus we find the instability growth rate from Eq. (28) as

$$
s(t) \propto \frac{k(t)}{\sqrt{h(t)}} \propto k_{0} q^{-1 / 4} t^{-3 / 4},
$$

allowing us to estimate the amplitude of disturbance as

$$
\delta h(t) \propto \exp \left(\int s(t) d t\right) \propto \exp \left(k_{0} q^{-1 / 4} t^{1 / 4}\right),
$$

in agreement with Eq. (49). Note that if $h(z) \sim z^{-\xi}$ and thus $v(z) \sim z^{2 \xi}$, the thread would be stable if $2 / 7<\xi<1 / 2$. This could be realized if the gravitational field increased as $g(z)$ $\sim z^{\zeta}$ with $\zeta=4 \xi-1$, so the case of constant $g(\xi=1 / 4)$ is only slightly below the limit of stability $(\xi=2 / 7)$.

The fact that our asymptotic results are independent of viscosity is quite counterintuitive. Our everyday experience tells us that only very viscous fluids form long threads, whereas low-viscosity fluids like water are very vulnerable to the Rayleigh-Plateau instability and drop formation. Viscous effects in Eqs. (36) and (37) asymptotically delay the instability, but they only seem to produce mild corrections $\propto \exp \left(-\Gamma k t^{-1 / 4}\right)$ and cannot account for the drastic differences between, say, water and syrup. The proper treatment of the role of viscosity in the instability of a falling thread goes beyond our asymptotic analysis, since the drop formation in less viscous fluids presumably happens before the asymptotic state (14) has had time to form. How this happens remains an open question which we hope to address in future research.

\section{DISCUSSION}

The stationary flow of a long falling viscous thread has the asymptotic shape $h(z) \sim z^{-1 / 4}$ depending neither on surface tension $\sigma$ nor viscosity $\nu$. Nearer the outlet the stationary shape depends on surface tension and viscosity and $h(z) \sim z^{-1}$. The crossover length between the viscous and inertial domains scales like $\nu^{2 / 3} \sigma^{-1 / 2}$. The numerical solution of the stationary problem fits well the experimentally observed shapes.

The asymptotic shape $h(z) \sim z^{-1 / 4}$ is unstable as expected from the classical results for the stability of a fluid cylinder. The perturbations grow, however, very slowly, such that the Fourier components increase asymptotically only as $\exp \left(\right.$ const $\left.\times t^{1 / 4}\right)$, where the constant is independent of viscosity. The instability is so weak that if the gravitational field was increasing slowly-i.e., $g(z) \sim z^{\zeta}$, with $1 / 7<\zeta<1$ - the thread would become asymptotically stable.

\section{ACKNOWLEDGMENTS}

We would like to thank Jens Eggers for helpful advice and several important discussions. We are grateful to Sid Nagel for several inspiring discussions and to Christophe Clanet for helpful comments. T.B. thanks the Danish Natural Science Research Council for support. After the submission of this manuscript, we became aware of a manuscript before publication by U. S. Sauter and H. W. Buggisch, where some of the results in Sec. III were obtained independently. We thank the authors for providing us with this manuscript.

\section{APPENDIX}

In this appendix we show that the explicit dependence on $x$ in Eq. (41) can be neglected when $t \gg|x|$. Neglecting this variation led to the "local" solution (49):

$$
\begin{gathered}
\tilde{b}_{0}(k, t)=t^{-1} \exp \left(\varepsilon k t^{1 / 4}\right), \\
\tilde{a}_{0}=\frac{2 i}{k} \tilde{b}_{0 t},
\end{gathered}
$$

where $\varepsilon=\sqrt{8} q^{-1 / 4}$ and where we define the direct and inverse Fourier transforms as

$$
\begin{gathered}
\widetilde{a}(k, t)=\frac{1}{2 \pi} \int a(x, t) \exp (-i k x) d x, \\
a(x, t)=\int \widetilde{a}(k, t) \exp (i k x) d k .
\end{gathered}
$$

Let us now include effects of nonlocality by the Fourier transformation of Eq. (41):

$$
\begin{aligned}
\tilde{a}_{t}+\frac{1}{2 \pi} \int \frac{2 a \exp (-i k x)}{x+t} d x= & q^{-1 / 2} \frac{1}{2 \pi} \int \frac{b_{x} \exp (-i k x)}{(x+t)^{3 / 2}} d x \\
& +q^{-1 / 2} \frac{1}{2 \pi} \int \frac{b \exp (-i k x)}{2(x+t)^{5 / 2}} d x
\end{aligned}
$$

We assume that $|x| \ll t$ and expand integral kernels in Eq. (A4) in a power series of $|x| / t$. First we consider LHS of Eq. (A4):

$$
\begin{aligned}
\frac{1}{2 \pi} \int \frac{2 a \exp (-i k x)}{x+t} d x= & 2 t^{-1} \widetilde{a}+2 \sum_{n=1}^{\infty} t^{-n-1} C_{-1}^{n} \frac{1}{2 \pi} \\
& \times \int x^{n} a \exp (-i k x) d x .
\end{aligned}
$$

We substitute

$$
\frac{1}{2 \pi} \int x^{n} a \exp (-i k x) d x=(i)^{n}\left(\frac{d}{d k}\right)^{n} \widetilde{a}
$$

and get 


$$
\frac{1}{2 \pi} \int \frac{2 a \exp (-i k x)}{x+t} d x=2 t^{-1} \tilde{a}+2 \sum_{n=1}^{\infty} t^{-n-1} C_{-1}^{n}(i)^{n}\left(\frac{d}{d k}\right)^{n} \tilde{a}
$$

where $C_{m}^{n}$ is a binomial coefficient. Let us estimate the correction term in the RHS of Eq. (A7) for the local solution (A1) and (A2). After some algebra we obtain

$$
\begin{aligned}
\frac{1}{2 \pi} \int \frac{2 a_{0}(t) \exp (i k x)}{x+t} d x= & 2 t^{-1} \widetilde{a}_{0}(t)+\widetilde{b}_{0}(t) \sum_{n=1}^{\infty} t^{-n} C_{-1}^{n}(i)^{n+1} \\
& \times \sum_{m=0}^{n} C_{n}^{m}(n-m) !(-1)^{m-1} k^{-1-n+m} \\
& \times \varepsilon^{m} t^{(m-7) / 4}\left[k \varepsilon+(m-4) t^{-1 / 4}\right] .
\end{aligned}
$$

Thus the highest-order term under the inner summation is proportional to $t^{(n-7) / 4}$. Taking into account the general multiplier $t^{-n-1}$, we have a leading-order correction proportional to $t^{-(3 n+7) / 4} t^{-1} \exp \left(\varepsilon k t^{1 / 4}\right)$. This should be compared with the main contribution on the RHS. of Eq. (A4)-i.e., $t^{-3 / 2} t^{-1} \exp \left(\varepsilon k t^{1 / 4}\right)$. Obviously, for $n \geqslant 1$ at $t \mapsto \infty$ we have $t^{-(3 n+7) / 4}<t^{-3 / 2}$ and we conclude that all terms produced by LHS of Eq. (A5) are subdominant.

Now we continue with the correction terms on the RHS. of Eq. (A4). Applying the same method, we get

$$
\frac{1}{2 \pi} \int \frac{b_{x} \exp (-i k x)}{(x+t)^{3 / 2}} d x=t^{-3 / 2} \sum_{n=0}^{\infty} t^{-n} C_{-3 / 2}^{n}(i)^{n+1}\left(\frac{d}{d k}\right)^{n}(k \widetilde{b}) .
$$

So the correction terms $(n \geqslant 1)$ are

$$
\propto t^{-3 / 2} \sum_{n=1}^{\infty} t^{-3 n / 4} C_{-3 / 2}^{n}(i)^{n+1} \varepsilon^{n-1}\left[k \varepsilon+n t^{-1 / 4}\right] \tilde{b}_{0} .
$$

Now the leading-order term is proportional to $t^{-3 n / 4} t^{-3 / 2} \widetilde{b}_{0}(t)$, which is dominated by the main term proportional to $t^{-3 / 2} \widetilde{b}_{0}(t)$ when $t \rightarrow \infty$. As for the last term in the RHS of Eq. (A4), it only produces minor corrections to the main solution even in the leading order. Thus we see that for $|x| / t \ll 1$ we can neglect the effects of nonlocality in Eq. (A4).
[1] S. Chandrasekhar, Hydrodynamic and Hydromagnetic Stability (Dover, New York, 1981).

[2] J. Eggers, Rev. Mod. Phys. 69, 865 (1997).

[3] L. D. Landau and E. M. Lifshitz, Fluid Mechanics (Pergamon, Oxford, 1984).

[4] J. Eggers and T. F. Dupont, J. Fluid Mech. 262, 205 (1994).

[5] N. S. Clarke, Q. J. Mech. Appl. Math. 22, 247 (1968).

[6] A. Kaye and D. G. Vale, Rheol. Acta 8, 1 (1969).

[7] E. O. Tuck, J. Fluid Mech. 76, 625 (1976).

[8] J. Geer, Phys. Fluids 20, 1613 (1977).
[9] J. Geer, Phys. Fluids 20, 1622 (1977).

[10] J. Geer and J. C. Strikwerda J. Fluid Mech. 135, 155 (1983).

[11] S. Senchenko, Ph.D. thesis, DTU, Denmark, 2004.

[12] C. M. Bender and S. A. Orszag, Advanced Mathematical Methods for Scientists and Engineers (McGraw-Hill, New York, 1978).

[13] M. P. Brenner, X. D. Shi, and S. R. Nagel, Phys. Rev. Lett. 73, 3391 (1994).

[14] S. Tomotika, Proc. R. Soc. London, Ser. A 153, 302 (1936). 